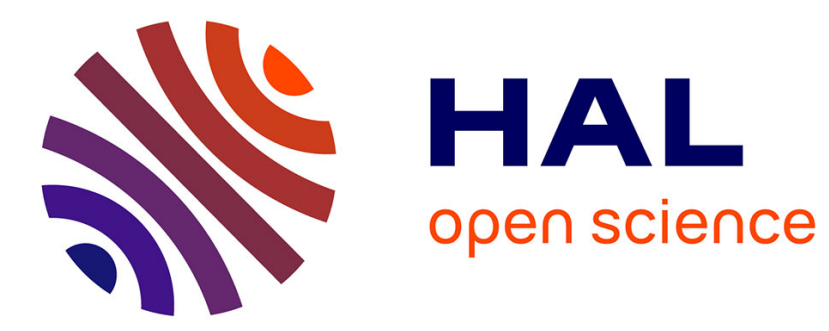

\title{
Micromechanical modeling for the probabilistic failure prediction of stents in high-cycle fatigue
}

\author{
Raphael Guerchais, G. Scalet, A. Constantinescu, F. Auricchio
}

\section{To cite this version:}

Raphael Guerchais, G. Scalet, A. Constantinescu, F. Auricchio. Micromechanical modeling for the probabilistic failure prediction of stents in high-cycle fatigue. International Journal of Fatigue, 2016, 87, pp.405-417. 10.1016/j.ijfatigue.2016.02.026 . hal-01392531

\section{HAL Id: hal-01392531 \\ https://hal.science/hal-01392531}

Submitted on 4 Nov 2016

HAL is a multi-disciplinary open access archive for the deposit and dissemination of scientific research documents, whether they are published or not. The documents may come from teaching and research institutions in France or abroad, or from public or private research centers.
L'archive ouverte pluridisciplinaire HAL, est destinée au dépôt et à la diffusion de documents scientifiques de niveau recherche, publiés ou non, émanant des établissements d'enseignement et de recherche français ou étrangers, des laboratoires publics ou privés. 


\title{
Micromechanical modeling for the probabilistic failure prediction of stents in high-cycle fatigue
}

\author{
R. Guerchais ${ }^{\mathrm{a}, *}$, G. Scalet ${ }^{\mathrm{a}}$, A. Constantinescu ${ }^{\mathrm{a}}$, F. Auricchio ${ }^{\mathrm{b}}$ \\ a Laboratoire de Mécanique des Solides, École Polytechnique, CNRS UMR7649, Université Paris-Saclay, 91128 Palaiseau Cedex, France \\ ${ }^{\mathrm{b}}$ Dipartimento di Ingegneria Civile e Architettura, Università di Pavia, Via Ferrata 3, 27100 Pavia, Italy
}

Keywords:

Balloon-expandable stent

Crystal plasticity

High-cycle fatigue

Micromechanics

Probabilistic failure prediction

\begin{abstract}
A B S T R A C T
The present paper introduces a methodology for the high-cycle fatigue design of balloon-expandable stents. The proposed approach is based on a micromechanical model coupled with a probabilistic methodology for the failure prediction of stents. This allows to account for material heterogeneity and fatigue scatter, to introduce a fatigue criterion able to consider stress gradients, and to perform a probabilistic analysis to obtain general predictions from a limited number of realizations of microstructures investigated. Numerical simulations have allowed to highlight the noteworthy characteristics of the mechanical response in the stent as well as the heterogeneity of the mechanical fields due to stress concentrations in the unit cell geometry and to strain incompatibilities between the grains induced by the anisotropy of their mechanical behavior. The predicted survival probability of the stent is in accordance with the experimental data from the literature. Moreover, the influence of the amplitude of the arterial pressure on the fatigue strength of the stent has been evaluated.
\end{abstract}

\section{Introduction}

Stenting intervention has been largely exploited in the treatment of cardiovascular diseases which represent the leading cause of death and illness accounting for $30 \%$ of the deaths worldwide annually [1]. Stents are small tube-like devices used to sustain narrowed or weakened arteries. The success of stenting is partly due to minimally-invasive procedures like the balloon-expandable coronary stents employed to prevent coronary restenosis after angioplasty.

Recently, stent failure has emerged as a major concern within the clinical and engineering community [2]. From the clinical point of view, failure of stents may lead to restenosis, thrombosis, pseudoaneurysm formation, and embolization, resulting in both shortand long-term morbidity and mortality [3]. From a mechanical point of view, failure of stents can be due to static loading during deployment or to cyclic loading caused by pulsatile blood pressure, bending, torsion, tension, and compression, due to the movement and muscle contractions of the patient [4]. The factors affecting the fatigue performance of stents are, on the one hand, the material properties, the design and the manufacturing of the stent and, on the other hand, the specificities of the patient as his anatomy and his lifestyle.

Stents are manufactured either through welding of microscopic wires or through laser cutting from thin-walled tubes. The characteristic dimensions of the repeated unit cell play distinct functions within the deformation of the stent and can enhance its specific performance. The final stent structure of both manufacturing procedures is a truss lattice composed of struts connected by hinges, with cross-sections in the range of $50-150 \mu \mathrm{m}$ and small radii, affecting stent performance due to high stress concentrations $[5,1,6]$.

Given the small scale of such devices and the complex loading conditions combining both hard and soft materials (the metallic stent and the blood vessel respectively), experimental measurements are difficult to perform, time-consuming and expensive, especially considering that current regulatory bodies require or recommend at least a ten-year life for stents, that corresponds to $4 \cdot 10^{8}$ systolic-diastolic pulsatile cycles $[7,8]$. Therefore, computer-based modeling is now used as a cost-effective tool for providing useful insight concerning the prediction of stent performance; see Ref. [9] for a review on fatigue of metallic stents. Numerical procedures, combined with experimental investigations, allow to make lifesaving decisions and to possibly improve both the clinical procedure and stent design. Several studies exploring, investigating and inquiring the numerical fatigue-life assessment of balloonexpandable stents have been reported in the literature [10-21]. 
However, the larger number of analyses are not matched with experimental data as a complete validation method would require [22].

Moreover, the proposed fatigue prediction methodologies generally neglect the inhomogeneous nature of the microstructure $[11,13-16,21]$ and although they may provide a practical and relevant answer to the fatigue design of stents, they cannot predict the scatter of the fatigue strength which might be induced by this heterogeneity [18]. Indeed, in high-cycle fatigue, the crack initiation is intimately related to localization of the plastic slip which strongly depends on the crystallographic orientations of the grains. Moreover, given the small size of stents with respect to the mean grain size and thus, the lack of representativeness of the crystalline orientations in the vicinity of the critical regions of stents, the fatigue strength can significantly vary from one crystalline orientation configuration to another.

The micromechanical framework introduced, in the context of fatigue analysis of stents, by Sweeney et al. [17-20] offers new perspectives as it enables to model the anisotropic elastic-plastic crystals and to define their orientations. However, the fatigue criteria proposed by the authors may not be able to accurately predict the fatigue behavior of stents. Indeed, the presence of small curvature radii in stent struts are responsible for high stress concentrations. The resulting stress gradients in the structure have a beneficial effect on the fatigue strength which requires the adoption of specific approaches (e.g. the theory of critical distance [14], volumetric approaches [23]) to be predicted.

The present work aims to provide answers to some outstanding issues and especially concerning the effect of the lack of representativeness of the crystalline orientations on the scatter of the fatigue strength. To this purpose, we introduce a micromechanical model coupled with a probabilistic methodology for the failure prediction of stents. This allows to (i) account for material heterogeneity and thus the scatter in fatigue due to the variability of crystalline orientations, (ii) introduce a fatigue criterion able to consider stress gradients, and (iii) perform a probabilistic analysis to obtain general predictions from a limited number of realizations of microstructures investigated.

Due to the complexity of the problem (large number of degrees of freedom, non-linearities due to the constitutive model and the contact between the stent and the artery) and in order to achieve reasonable computation times, the numerical analysis conducted in the present work is divided in two steps, analogous to the methodology proposed by Sweeney et al. [17-20]. Firstly, a finite element analysis (FEA) modeling the stent deployment and fatigue in an artery is conducted, using a complete three-dimensional stent and a homogeneous elasto-plastic model. Secondly, the results acquired from this simulation provide an estimation of the boundary conditions which have to be applied in a simplified polycrystalline model of a fraction of the stent. This analysis has the purpose of determining the elastic shakedown mechanical state of the stent at the grain scale. Then, a probabilistic analysis, relying on a fatigue criterion sensitive to the microstructure and based on the weakest link hypothesis, is conducted in order to estimate the survival probability of the stent. The predictions obtained are compared to the results of the fatigue tests conducted on $316 \mathrm{~L}$ austenitic steel stents by Kapnisis et al. [24] in order to assess the predictive ability of the fatigue criterion.

The present paper is organized as follows. Section 2 presents the modeling of both the homogeneous and the polycrystalline stent. Section 3 describes the mechanical responses of the polycrystalline stent. Then, Section 4 proposes a fatigue criterion that is relevant with respect to the specificities of the mechanical responses. In Section 5 a probabilistic analysis, relying on the fatigue criterion, is conducted in order to estimate the survival probability of the stent. Finally, comments and conclusions are presented in Section 6.

\section{Modeling}

The present work aims to model the experiment proposed by Kapnisis et al. [24], an accelerated pulsatile durability test on Cypher $^{\circledR}$ stents made of 316L stainless steel and inserted in a straight mock artery made of natural rubber latex.

The particular choice of the stenting configuration does not restrain the generality of the present method and has been chosen only for the purpose of illustration. A crystal plasticity finite element model of a complete three-dimensional stent would lead to use a complex microstructure containing approximately 100,000 grains and would conduct to prohibitive computational time and resources.

As a consequence, we adopt simplifying modeling assumptions, similar to the ones adopted by Sweeney et al. [17]. The analysis will start with a three-dimensional analysis of a homogeneous stent which will provide the boundary conditions to apply in a two-dimensional "unit cell" of the stent with a crystal plasticity material behavior (see Fig. 1).

\subsection{Three-dimensional homogeneous model}

The three-dimensional homogeneous model consists of the stent, the surrounding vessel and a balloon (see Fig. 2a) and is described next. The mechanical analysis is performed with the FE method, using the software Abaqus/Standard (Simulia, Dassault Systèmes, Providence, RI, USA).

Stent. The Cypher ${ }^{\circledR}$ stent has an inner diameter $D_{s, i}=0.85 \mathrm{~mm}$, an outer diameter $D_{s, o}=1.15 \mathrm{~mm}$ and a length $L_{s}=8.4 \mathrm{~mm}$ in the initial undeformed configuration. The strut pattern is constituted by 36 complete unit cells in addition to 12 truncated unit cells located at the extremities. The mesh is defined by 31,848 nodes and 17,280 three-dimensional linear brick elements.

The linear elastic component of the material behavior is described by the Young's modulus $E$ and the Poisson's ratio $v$. The time-independent plasticity model is defined by a von Mises yield surface:

$f=\sqrt{\frac{3}{2}\left(\underline{\underline{\sigma}}^{d}-\underline{\underline{X}}^{d}\right):\left(\underline{\underline{\sigma}}^{d}-\underline{\underline{X}}^{d}\right)}-\sigma^{y}-R$

where $\sigma^{y}, \underline{\underline{\sigma}}, \underline{\underline{X}}$ and $R$ denote the initial yield stress, the stress tensor, the backstress tensor and the isotropic hardening variable, respectively. The superscript ${ }^{d}$ designates the deviatoric part of the tensor.

The non-linear evolution of the isotropic hardening variable $R$ is given by Voce's law:

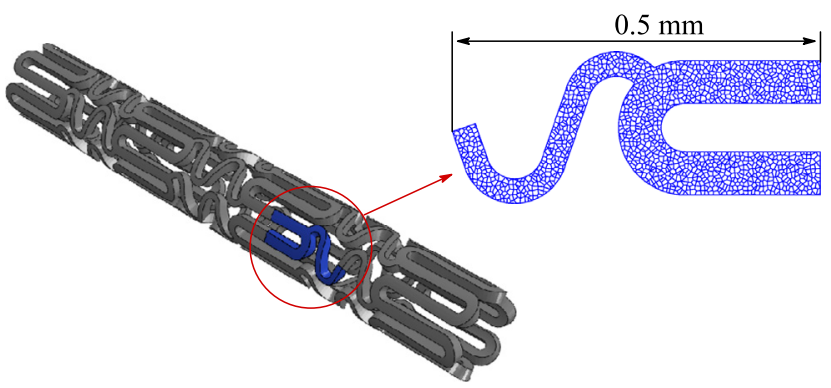

Fig. 1. Geometries of the three-dimensional Cypher ${ }^{\circledR}$ stent (the unit cell is highlighted in blue) and the two-dimensional unrolled polycrystalline unit cell. (For interpretation of the references to colour in this figure legend, the reader is referred to the web version of this article.) 


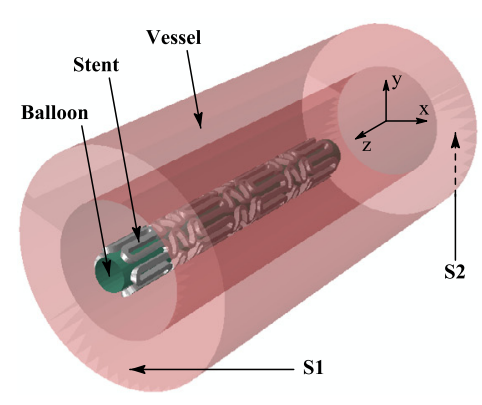

(a)

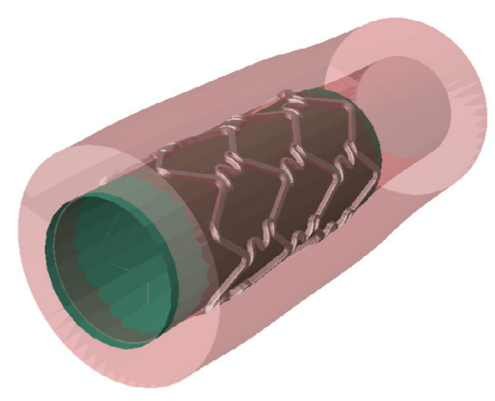

(b)

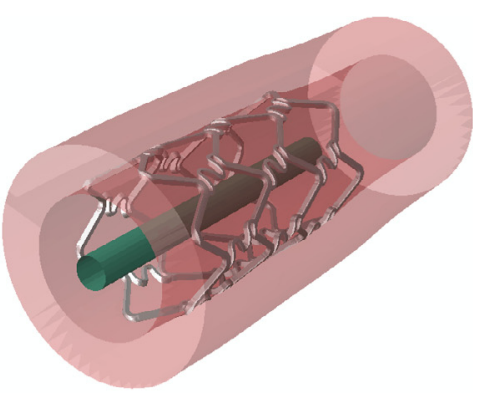

(c)

Fig. 2. Geometry of the three-dimensional FE model: (a) in the initial configuration, (b) after stent deployment, and (c) after stent recoil.

$\dot{R}=B\left(Q_{\infty}-R\right) \dot{p}$

where $p$ is the equivalent plastic strain and $Q_{\infty}$ and $B$ are material parameters.

The Armstrong-Frederick's law describes the non-linear evolution of the backstress tensor:

$\underline{\underline{X}}=\frac{2}{3} C \underline{\underline{\varepsilon}}^{p}-\gamma \underline{\underline{X}} \dot{p}$

where $C$ and $\gamma$ are material parameters and $\underline{\underline{\varepsilon^{p}}}$ denotes the plastic strain tensor.

The model parameters have been calibrated for a $316 \mathrm{~L}$ biocompatible steel [25] using the experimental data obtained from a monotonic tensile test and low-cycle fatigue tests in several loading conditions: tension, torsion and combined out-of-phase tension and torsion. These parameters are summarized in Table 1.

Vessel. In the initial configuration, the vessel is modeled as thick cylindrical tube with inner diameter $D_{v, i}=2.5 \mathrm{~mm}$, outer diameter $D_{v, o}=3.5 \mathrm{~mm}$, and a length of $L_{v}=12 \mathrm{~mm}$. The mesh consists of 11,700 hybrid three-dimensional linear brick elements and 15,808 nodes.

Its mechanical behavior is described by the Mooney-Rivlin hyper-elastic model which is based on the following strain energy potential:

$U=C_{10}\left(\bar{I}_{1}-3\right)+C_{01}\left(\bar{I}_{2}-3\right)$

where $C_{10}$ and $C_{01}$ are temperature-dependent material parameters taken from [26] and summarized in Table $1 . \bar{I}_{1}$ and $\bar{I}_{2}$ denote the first and second strain invariants defined as:

$\bar{I}_{1}=\bar{\lambda}_{1}^{2}+\bar{\lambda}_{2}^{2}+\bar{\lambda}_{3}^{2}$ and $\bar{I}_{2}=\bar{\lambda}_{1}^{(-2)}+\bar{\lambda}_{2}^{(-2)}+\bar{\lambda}_{3}^{(-2)}$

as functions of the deviatoric stretches $\bar{\lambda}_{i}=J^{-1 / 3} \lambda_{i}$ with $J$ the total volume ratio and $\lambda_{i}$ the principal stretches.

Balloon. The balloon is a cylindrical surface with a diameter $D_{c}=0.8 \mathrm{~mm}$ in the initial configuration, discretized by 18864 node quadrilateral surface elements and 1909 nodes and will be used to impose a displacement driven inflation during the deployment of the stent.

\section{Loading steps.}

The mechanical analysis of the three-dimensional model is divided into four loading steps:
1. stretching and pressurization of the vessel;

2. stent deployment (see Fig. 2b);

3. stent recoil (see Fig. 2c);

4. diastolic-systolic cyclic loading, i.e. fatigue loading.

In the first step, the vessel is stretched by an axial displacement $U_{z}=1.2 \mathrm{~mm}$ on the planar surface S2 (see Fig. 2a), leading to an axial extension ratio $e_{z}=U_{z} / L_{v}=10 \%$. Additionally, a static pressure $P=13 \mathrm{kPa}(100 \mathrm{mmHg})$ is applied on the inner surface of the vessel. While stretching is always maintained, the static pressure is replaced by a cyclic diastolic-systolic pressure $P_{\text {art }}$ during the last step of the analysis. Furthermore, axial and circumferential constraints are applied to prevent rigid body motions.

The second step, the stent deployment induced by the inflation of the balloon, is modeled by increasing its diameter from $0.8 \mathrm{~mm}$ to $3.45 \mathrm{~mm}$ and imposing a friction-less contact between the balloon and the vessel. The third step, which represents the stent recoil, is modeled by deactivating the contact between the balloon and the stent and maintaining the contact between the stent and the vessel.

The final step, representing the diastolic-systolic cyclic loading, corresponds to the service loading and is responsible for the fatigue damage in the stent. It is carried out by applying a periodic pressure $P_{\text {art }}$ on the inner surface of the vessel. The pressure is defined by a triangle variation of amplitude $P_{a}=6 \mathrm{kPa}$ and mean $P_{m}=14 \mathrm{kPa}$. The minimum pressure, denoted as diastolic pressure, is $P_{\min }=8 \mathrm{kPa}(60 \mathrm{mmHg})$, while the maximum pressure, also denoted as systolic pressure, $P_{\max }=20 \mathrm{kPa}(160 \mathrm{mmHg})$, corresponds to the values used in the experiments [24]. Under the given loading conditions, four loading cycles were enough to reach an elastic shakedown state in the structure.

\subsection{Two-dimensional polycrystalline stent unit cell}

\subsubsection{Characteristics of the $316 \mathrm{~L}$ austenitic stainless steel}

The 316L steel considered here, used in biomedical applications, was the subject of an extensive characterization in high-cycle fatigue [27]. Its chemical composition is designed to provide a ferritefree microstructure and is summarized in Table 2. Moreover, martensitic transformations, occurring during heat treatment or mechanical loading, are prevented due to the high nickel percent-

Table 1

Material parameters used in the FEA of the three-dimensional homogeneous model.

\begin{tabular}{|c|c|c|c|c|c|c|c|c|}
\hline \multicolumn{2}{|l|}{ Vessel } & \multicolumn{7}{|l|}{ Stent } \\
\hline$C_{10}$ & $C_{01}$ & $E(\mathrm{GPa})$ & $v$ & $\sigma^{y}(\mathrm{MPa})$ & $Q_{\infty}(\mathrm{MPa})$ & $B$ & $C(\mathrm{MPa})$ & $\gamma$ \\
\hline $1.815 \cdot 10^{-1}$ & $6.506 \cdot 10^{-3}$ & 194 & 0.284 & 129 & 142 & 6.88 & $7.05 \cdot 10^{5}$ & $3.08 \cdot 10^{3}$ \\
\hline
\end{tabular}


Table 2

Chemical composition of the $316 \mathrm{~L}$ austenitic steel.

\begin{tabular}{llllll}
\hline Elements & $\mathrm{C}$ & $\mathrm{Cr}$ & $\mathrm{Ni}$ & $\mathrm{Mo}$ & $\mathrm{Mn}$ \\
\hline $\mathrm{Wt} \%$ & 0.02 & 19.0 & 15.0 & 3.0 & 1.9 \\
\hline
\end{tabular}

age. Therefore, the material is a purely austenitic structure, consisting of face-centered cubic crystals.

The grains, as observed thanks to electron backscatter diffraction (EBSD) analyses, have almost equal principal axes. The mean grain size is approximately equal to $14 \mu \mathrm{m}$ when defined as the square root of the mean area of the grain and including the twin boundaries. Two types of twin boundaries are observed in the microstructure: the $\Sigma 3$ twin boundary, which correspond to a misorientation of $60^{\circ}$ about the $\langle 111\rangle$ axis and the $\Sigma 9$ twin boundary, which can be described by a misorientation of $38.94^{\circ}$ about the $\langle 110\rangle$ axis. The Friedel index $\Sigma$ associated to the boundary corresponds to the inverse of the fraction of the coincidence sites. The analyses have revealed a large fraction of $\Sigma 3$ twin boundaries (approximately 50\%) and a small fraction of $\Sigma 9$ twin boundaries (approximately $2 \%$ ). The remaining boundaries are low angle grain boundaries (i.e. grain boundaries with a disorientation $\Delta \theta \leqslant 15^{\circ}$ ) or general grain boundaries, i.e. high angle grain boundary $\left(\Delta \theta>15^{\circ}\right)$ with high values of Friedel index $(\Sigma>27)$. These peculiarities are illustrated in Fig. 3 using an image quality map and an inverse pole figure map in which general and low angle grain boundaries are represented with black lines whereas $\Sigma 3$ and $\Sigma 9$ twin boundaries are represented with red and yellow lines respectively.

For the sake of simplicity, the twin boundaries are not described in the next finite element model of the polycrystalline unit cell of the stent. It can be noticed that this modeling choice has some consequences. Indeed, according to a numerical study conducted by Castelluccio and McDowell [28], larger values of a fatigue indicator parameter are observed in polycrystals containing twin boundaries than in microstructures without twins. The authors conclude that even though models without explicit consideration of twins can accurately estimate the fatigue life, they may not predict satisfactorily the location of the fatigue crack initiation. Thus, the fatigue design methodology proposed in the present work will not attempt to precisely determine the site of the crack initiation but only to assess the fatigue strength.

\subsubsection{Single crystal constitutive model}

It is now well-known that the anisotropic elasto-plastic behavior of single crystals is significantly related to the atomic arrangements in the crystalline lattice. In the case of austenitic steel, the atomic lattice is face-centered cubic (FCC) and consequently the elastic behavior has cubic symmetry and the plastic slip occurs along the [110] directions of $\{111\}$ planes, which correspond to closed-packed planes and directions of the crystalline lattice.

The linear elastic behavior with cubic symmetry is completely characterized by the following three coefficients: $C_{1111}, C_{1122}$ and $C_{1212}$ of the elastic tensor, where the coordinate axes are oriented by the crystal. The values used here originate from a study of Teklu et al. [29] and correspond to an austenitic Fe-18Cr-14Ni steel.

The nonlinear behavior of the single crystals is represented by the viscoplastic model proposed by Méric et al. [30]. In this model, the plastic slip rate $\dot{\gamma}_{s}$ of the slip system $s$ is described by a Nortontype flow rule:

$\dot{\gamma}_{s}=\left\langle\frac{\left|\tau_{s}-\chi_{s}\right|-r_{0}-r_{s}}{K}\right\rangle_{+}^{n} \operatorname{sgn}\left(\tau_{s}-\chi_{s}\right)=\dot{v}_{s} \operatorname{sgn}\left(\tau_{s}-\chi_{s}\right)$

The flow depends on the resolved shear stress $\tau_{s}$ acting on $s$ and the isotropic and kinematic hardening variables $r_{s}$ and $\chi_{s}$. K and $n$ are the viscosity parameters and $r_{0}$ is the critical resolved shear stress.

The resolved shear stress $\tau_{s}$ acting on $s$ is the contraction of the stress tensor $\underline{\underline{\sigma}}$ with the orientation tensor $\underline{\underline{m}}_{s}$ associated to the slip system $s$ :

$\tau_{s}=\underline{\underline{m_{s}}}: \underline{\underline{\sigma}}$

The orientation tensor is further defined as:

$\underline{\underline{m}}=\frac{\left(\underline{n_{s}} \otimes \underline{l}_{s}+\underline{l}_{s} \otimes \underline{n}_{s}\right)}{2}$

where $\underline{n}_{s}$ and $\underline{l}_{s}$ denote the the unit vectors normal to the slip plane and characterizing the slip direction respectively.

The isotropic and kinematic hardening variables $r_{s}$ and $\chi_{s}$ are further characterized by the following evolution equations:

$r_{s}=Q \sum_{r} h_{s r}\left(1-e^{-b v_{r}}\right)$

$\dot{\chi}_{s}=c \dot{\gamma}_{s}-d \dot{v}_{s} \chi_{s}$

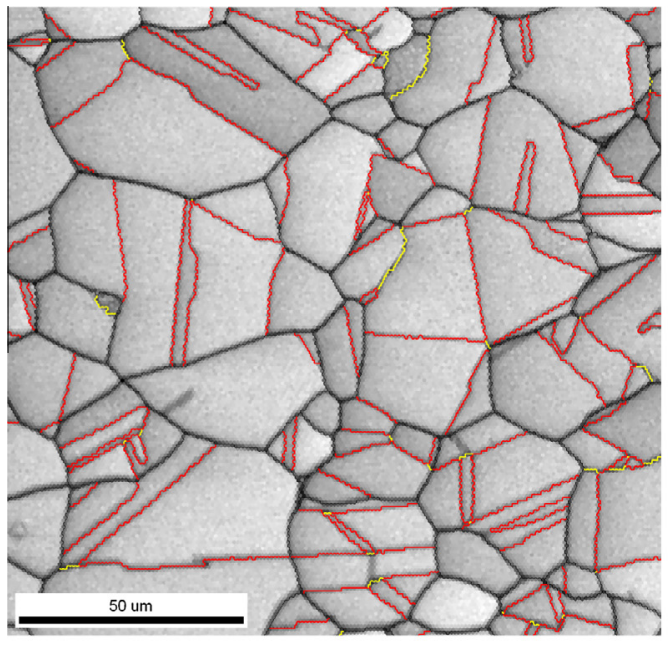

(a)

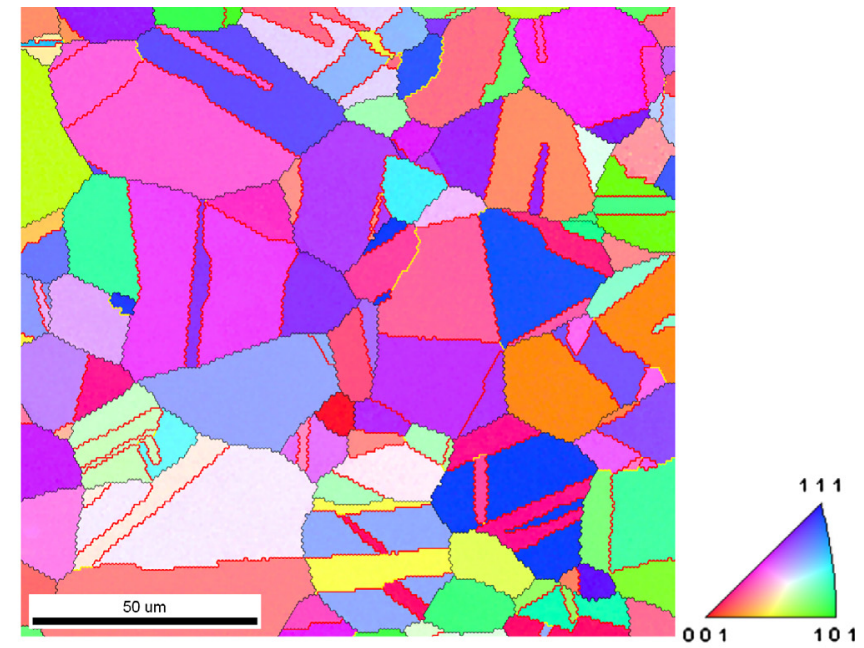

(b)

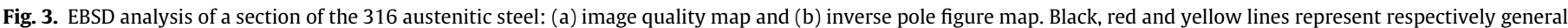

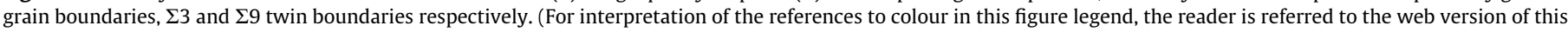
article.) 
In Eq. (9), the crossed influence of the accumulated plastic slip $v_{r}$ on the slip system $r$ on the hardening of the slip system $s$ is characterized by the $h_{s r}$ component of the interaction matrix, as proposed initially in [31]. Moreover, the interaction matrix permits to account for self-hardening, characterized by $h_{0}$, and for the latent hardening, described by $h_{1}, h_{2}, h_{3}, h_{4}$ and $h_{5}$. Each component of the later corresponds to a given type of slip system interaction: coplanar junction, collinear interaction, Lomer junction, glissile junction, Hirth lock. $Q, b$ and $c, d$ are further isotropic and kinematic hardening parameters respectively (Eq. (10)).

The material parameters of the single crystal viscoplaticity model have been calibrated for a 316L steel [25] using the experimental data obtained from a monotonic tensile test and low-cycle fatigue tests in several loading conditions: tension, torsion and combined out-of-phase tension and torsion. All material parameters are summarized in Table 3.

The orientation of each crystal of the polycrystalline aggregate is explicitly provided by defining the corresponding Euler angles with respect to the global coordinate system. Twenty independent selections of Euler angles, representative of a non-textured polycrystal, have been procedurally generated and employed in the finite element analysis. The generation of these sets of orientation was performed using the "hybrid integer approximation" method proposed by Eisenlohr and Roters [32].

\subsubsection{Geometry and mesh of the unit cell}

The two-dimensional domain $\Omega$ of the unit cell is defined by rolling out the external surface of the three-dimensional stent unit cell. The rolled out stent unit cell is then partitioned into subdomains, representing the grains of the polycristal, by a Voronoi tessellation.

This decomposition is constructed from a set $S$ of $N_{g}$ random points denoted as seeds. Each grain associated to a seed $p \in S$ is the subdomain defined by the set of points $x$ closer to the seed $p$ than to other seed $q \in S$ :

$\operatorname{Vor}_{\mathrm{S}}(p)=\{x \in \Omega \mid \forall q \in S,\|x-p\| \leqslant\|x-q\|\}$

In the present case, $\|x-p\|$ corresponds to the euclidean distance between the points $x$ and $p$.

The Voronoi tessellation, in a two-dimensional plane, leads to a subdivision delimited by convex polygons. A microstructure described with this tessellation can be understood as the result of specific condition of grain growth. More precisely, it implies that the growth from the seeds is isotropic, homogeneous and starts, at the same time, in all seeds. Moreover, grain growth in a direction stops when the grain boundary reaches either another grain boundary or the domain boundary.

The unit cell contains $N_{g}=1452$ random seeds which insured a mean grain size of approximately $14 \mu \mathrm{m}$, in accordance with microscopic observations. The Voronoi tesselation is obtained thanks to the sweepline algorithm proposed by Fortune [33]. The geometry is then regularized by deleting small edges and arcs of circles in order to avoid excessive mesh refinement.

Table 3

Material parameters used in the FEA of the polycrystalline stent.

\begin{tabular}{|c|c|c|c|c|c|c|c|}
\hline \multicolumn{3}{|c|}{ Cubic elasticity } & \multicolumn{2}{|l|}{ Viscosity } & \multicolumn{3}{|c|}{$\begin{array}{l}\text { Kinematic } \\
\text { hardening }\end{array}$} \\
\hline $\begin{array}{l}C_{1111} \\
(\mathrm{GPa})\end{array}$ & $\begin{array}{l}C_{1122} \\
(\mathrm{GPa})\end{array}$ & $\begin{array}{l}C_{1212} \\
(\mathrm{GPa})\end{array}$ & $K\left(\mathrm{MPa} \mathrm{s}^{1 / n}\right)$ & $n$ & $c(\mathrm{MPa})$ & & $d$ \\
\hline 198 & 125 & 122 & 10 & 10 & $2.04 .10^{5}$ & & $3.63 .10^{3}$ \\
\hline \multicolumn{8}{|c|}{ Isotropic hardening } \\
\hline$r_{0}(\mathrm{MPa})$ & $Q(\mathrm{MPa})$ & $b$ & $h_{0}$ & $h_{2}$ & $h_{3}$ & $h_{4}$ & $h_{5}$ \\
\hline 87.0 & 1.06 & 4.88 & 1 & 0.438 & 77.2 & 4.31 & 2.41 \\
\hline
\end{tabular}

An example of a unit cell is displayed in Fig. 4. The discretization of the geometry is carried out with the FE mesh generator Gmsh [34] using three-node triangular elements. Each grain is discretized in average with 23 elements and a generalized plane strain hypothesis is adopted. The finite element analysis is conducted using Zebulon software.

\subsubsection{Boundary conditions}

Because of the finite length of the stent, and despite the periodicity of the unit cell and the symmetries in the structure, nonperiodic mechanical solutions in the unit cells are expected. Moreover, the unit cells located at the extremities of the stent are truncated and thus, their mechanical responses should be different from those of the complete unit cells. However, thanks to the 6fold rotational symmetry, about the $z$-axis, of the structure and of the boundary conditions (see Fig. 2), the stent unit cells located in the same interval $\left[z_{\min }, z_{\max }\right]$ have the same mechanical response, thus decreasing the number of different mechanical responses from 48 to 8 . Moreover, these 8 mechanical responses are only slightly different. For example, the maximum value reached by the von Mises stress $\sigma_{V M \text { max }}$ for each unit cell is almost constant: the maximum difference, between the most and less critical unit cells in term of $\sigma_{V M, \max }$, is indeed inferior to $10 \%$ at the end of the cyclic loading. Thus, for the sake of simplicity and because the differences between the mechanical responses of the different unit cells are small, only the unit cell having the maximum value of the von Mises stress is studied and is used to define the boundary conditions applied to the polycrystalline unit cell.

Once the three-dimensional unit cell is selected, the coordinates $(x(t), y(t), z(t))$, expressed in the Cartesian coordinate system $\left\{\underline{e}_{x}, \underline{e}_{y}, \underline{e}_{z}\right\}$, are extracted for each node on the edges of the unit cell and for each time step $t$ of the analysis. The aforementioned edges, located on the outer surface of the unit cell, are highlighted in red in Fig. 5a. The coordinates of the nodes are then expressed, for each time step, in a cylindrical coordinate system $\left\{\underline{e}_{r}, \underline{e}_{\theta}, \underline{e}_{z}\right\}$ (see Fig. 5b) using the following coordinate transformation:

$r(t)=\sqrt{\left(x(t)-x_{\Omega}\right)^{2}+\left(y(t)-y_{\Omega}\right)^{2}}$ and $\theta(t)=\arctan \left(\frac{y(t)-y_{\Omega}}{x(t)-x_{\Omega}}\right)$

with $x_{\Omega}$ and $y_{\Omega}$ corresponding to the coordinates $x$ and $y$, in the Cartesian coordinate system $\left\{\underline{e}_{x}, \underline{e}_{y}, \underline{e}_{z}\right\}$, of the center $\Omega$ associated to the cylindrical coordinate system $\left\{\underline{e}_{r}, \underline{e}_{\theta}, \underline{e}_{z}\right\}$ and placed on the axis of the stent.

The coordinates $\left(x^{\prime}(t), y^{\prime}(t)\right)$, expressed in the Cartesian coordinate system $\left\{\underline{e}_{x^{\prime}}, \underline{e}_{y^{\prime}}\right\}$ associated to the two-dimensional unit cell (see Fig. 4), of each node can be determined thanks to the same rolling out transformation used to define the geometry of the two-dimensional unit cell:

$x^{\prime}(t)=z(t) \quad$ and $\quad y^{\prime}(t)=r(t) \theta(t)$

The resulting positions of the nodes, from the initial state at time $t_{0}$ to the end of the cyclic loading at time $t_{f}$, are illustrated in Fig. 6. In this figure, the edges, where the displacements are applied, are

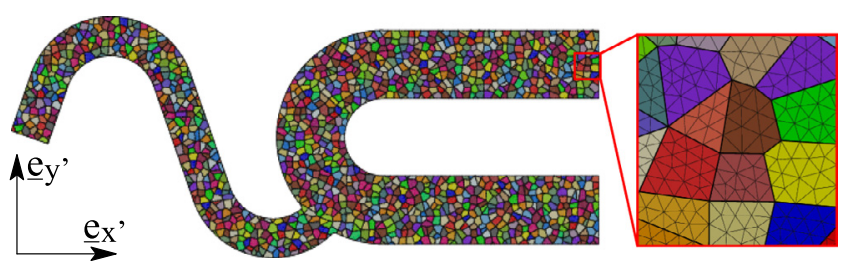

Fig. 4. Geometry and magnified view of the mesh of the 2-dimensional polycrystalline stent unit cell. 


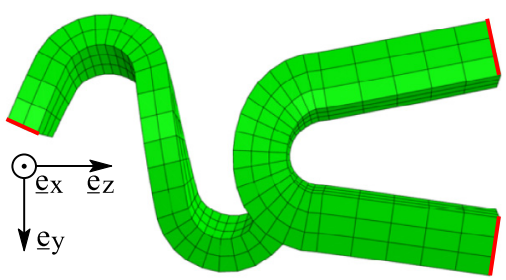

(a)

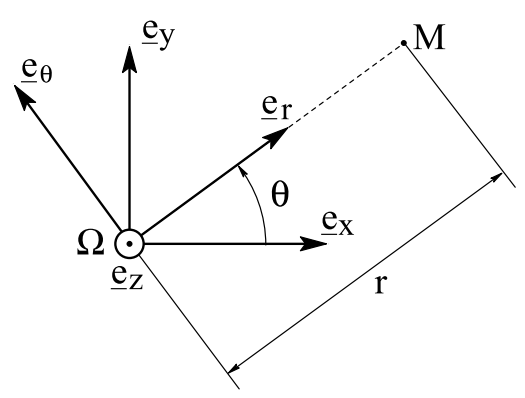

(b)

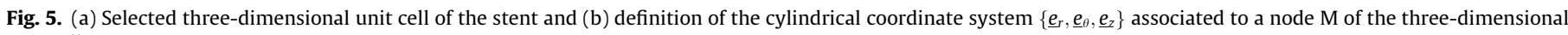
unit cell.

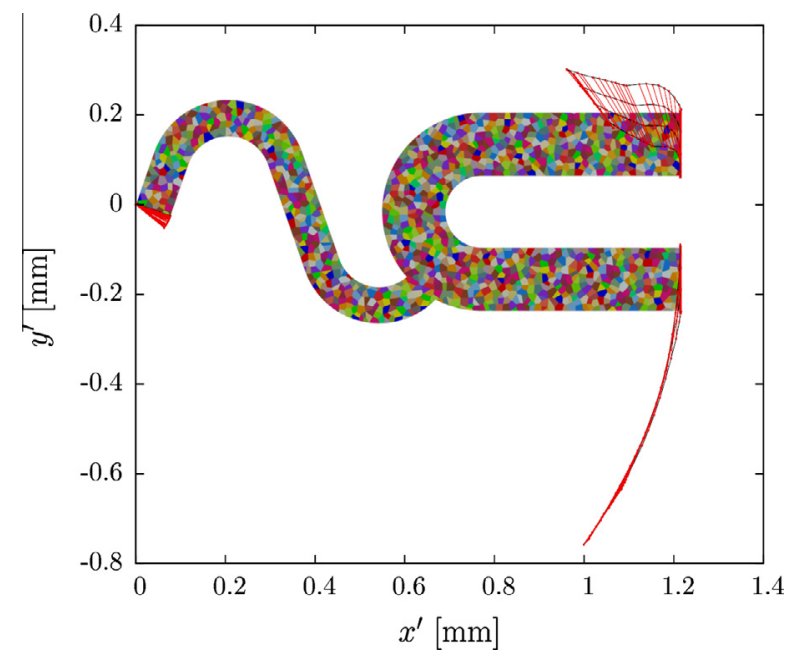

Fig. 6. Evolution of the position of the edges from the initial configuration to the cyclic loading (the red lines represent the edges at different time steps and the black lines represent the paths follows by the nodes of the 3D unit cell in the 2D plane). (For interpretation of the references to colour in this figure legend, the reader is referred to the web version of this article.)

represented by red lines; each line corresponding to the position of an edge at a given time step. The black lines represent the paths follow by the nodes of the three-dimensional unit cell in the twodimensional plane. It should be noted that the fatigue loading induced by the cyclic arterial pressure leads to small displacements of the edges in comparison to those observed during the stent deployment and the vessel deflation. This can be observed on the graph in Fig. 7 which represents the change of the spacing, along $\underline{e}_{y^{\prime}}$, between the two branches extremities of the unit cell $\Delta l_{y^{\prime}}(t)=l_{y^{\prime}}(t)-l_{y^{\prime}}\left(t_{0}\right)$ as a function of time $t$.

In order to define the boundary conditions applied on the edges of the polycrystalline stent unit cell, displacements with respect to the initial configuration at time $t_{0}$ have to be provided for each node belonging to the edges. The displacements $U_{x^{\prime}}$ and $U_{y^{\prime}}$, in the two-dimensional plane, of each selected node are computed as follows:

$U_{x^{\prime}}(t)=x^{\prime}(t)-x^{\prime}\left(t_{0}\right) \quad$ and $\quad U_{y^{\prime}}(t)=y^{\prime}(t)-y^{\prime}\left(t_{0}\right)$

Given that the edges of the 2D unit cell are more finely discretized than those of the three-dimensional unit cell, the values of displacements imposed on each node are linearly interpolated from the displacements obtained with the coarsely meshed three-dimensional unit cell.

\section{Analysis of the mechanical responses in the polycrystalline stent}

Before discussing about a methodology of fatigue design, the mechanical responses of the polycrystalline unit cells are analyzed in order to propose an appropriate fatigue criterion, able to correctly evaluate the fatigue strength of the structure.

In the first place, the distribution in the stent unit cell of local mechanical quantities are qualitatively analyzed in order to bring to the fore the main features of the mechanical responses of the polycrystalline stent unit cells. The discussion relies on Fig. 8 which represents two mechanical fields: the equivalent plastic slip (Fig. 8a) and the von Mises equivalent stress (Fig. 8b). These fields, which are determined at the gauss points at the end of the last
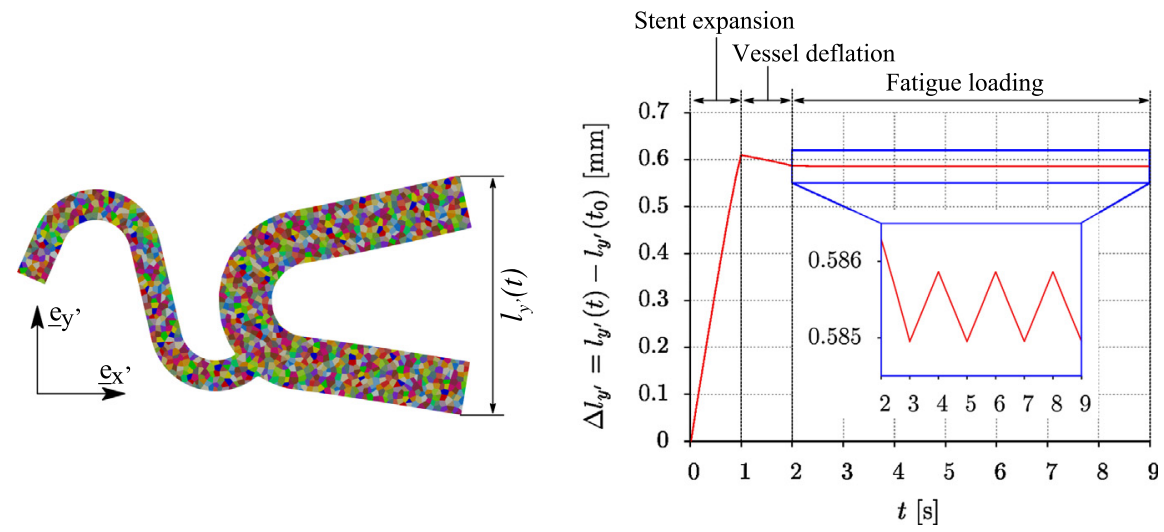

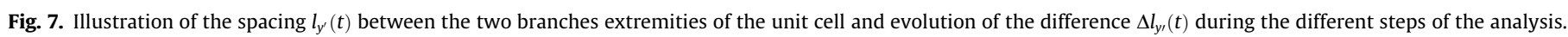



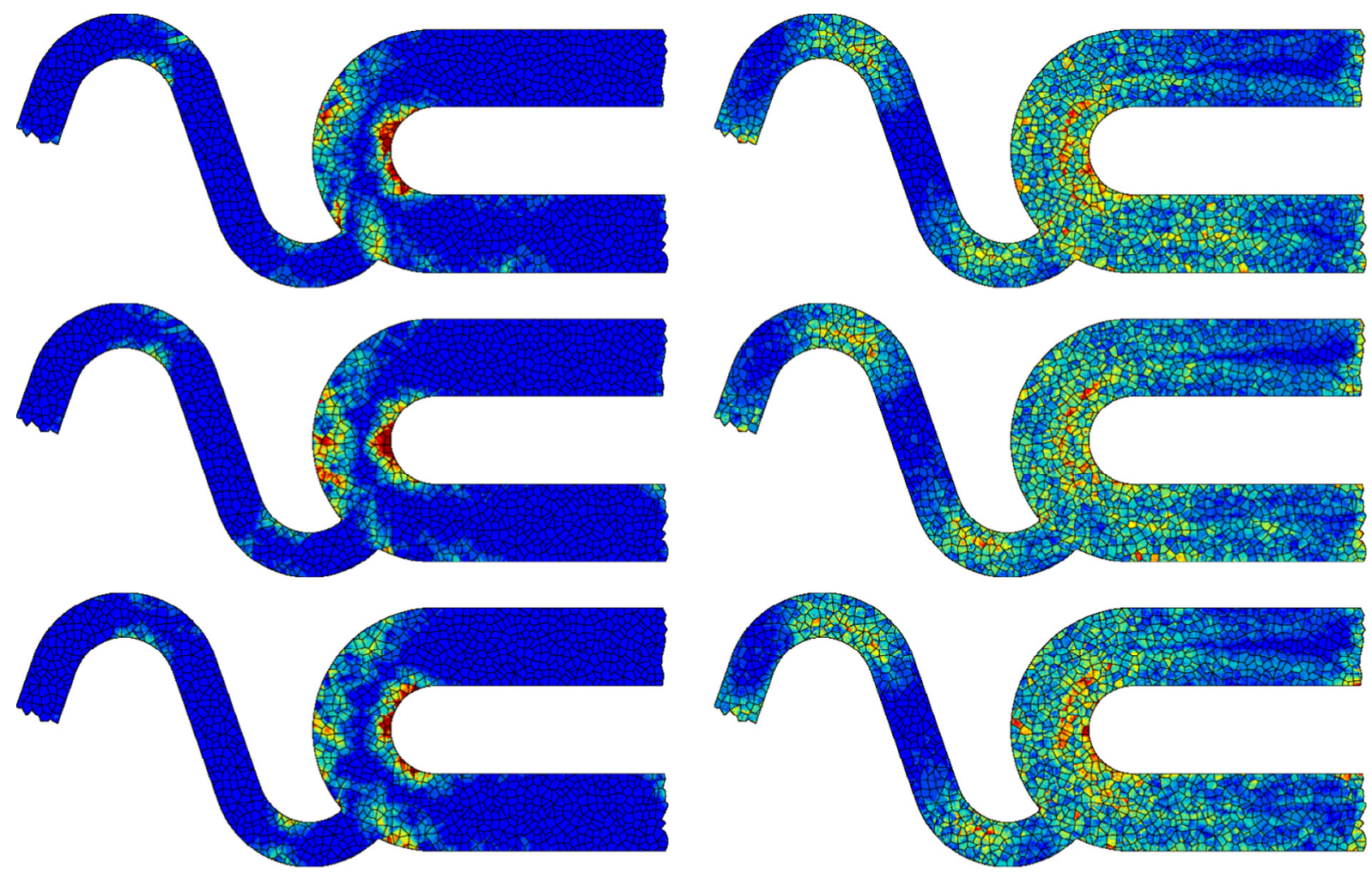

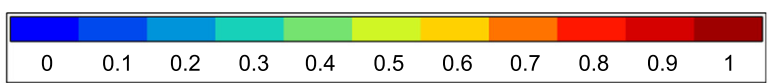

(a) Equivalent plastic slip

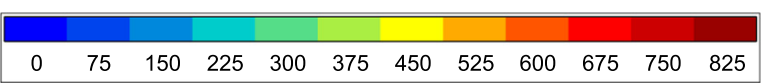

(b) Equivalent von Mises stress

Fig. 8. (a) Equivalent plastic slip and (b) von Mises equivalent stress fields in the stent unit cells for different sets of crystalline orientations.

loading cycle, correspond to the results obtained with three different set of crystalline orientations.

As it can be observed from this figure, both the equivalent plastic slip and the von Mises equivalent stress fields are heterogeneously distributed in the polycrystalline unit cells. More precisely, some regions exhibit a significant plastic activity or a high stress level whatever the crystalline orientations assigned to the grains. These "structural" heterogeneities are due to the loading conditions applied on the unit cell in addition to the complex geometry of the structure which contains several stress concentrations leading to significant gradients, in terms of stress or strain, in the unit cells. However, it is worth noting that the local mechanical responses present "microstructural" heterogeneities given that they still depend on the crystalline orientations assigned to the grains and on the anisotropy of the mechanical behavior. Indeed, the positions of the most plastified or stressed grains observed for a given orientation set are not strictly identical to those of the other unit cells. Moreover, the maximum values reached by the equivalent plastic slip and the von Mises equivalent stress vary from unit cell to unit cell due to the change of crystalline orientations. It should be noted that the possible configurations of crystalline orientations are responsible for the variability of the mechanical responses in the unit cells and thus are, at least partially, the cause of the scatter of the fatigue strength.

In the following, the mechanical responses of the polycrystalline unit cells are studied at the grain scale through the mesoscopic mechanical quantities, i.e. the quantities computed from the stress tensors averaged per grain $\langle\underline{\underline{\sigma}}\rangle_{g}$ which are obtained from the last loading cycle of the FE simulations. The distributions of three mechanical quantities, which are assumed to play a signif- icant part in the HCF failure, are discussed: the shear stress amplitude $\tau_{a}$, the normal stress amplitude $\sigma_{n, a}$, and the mean normal stress $\sigma_{n, m}$. Moreover, these distributions are compared to those observed in smooth microstructures subjected to simple loading conditions in order to determine to what extent the mechanical states in the unit cells differ from those obtained during classical fatigue tests.

To carry out this comparison, FEA representative of conventional fatigue tests are conducted on polycrystalline aggregates. The global geometry of the microstructure is smooth, i.e. without any stress concentrations (see Fig. 9). The Voronoi tessellation, pre-

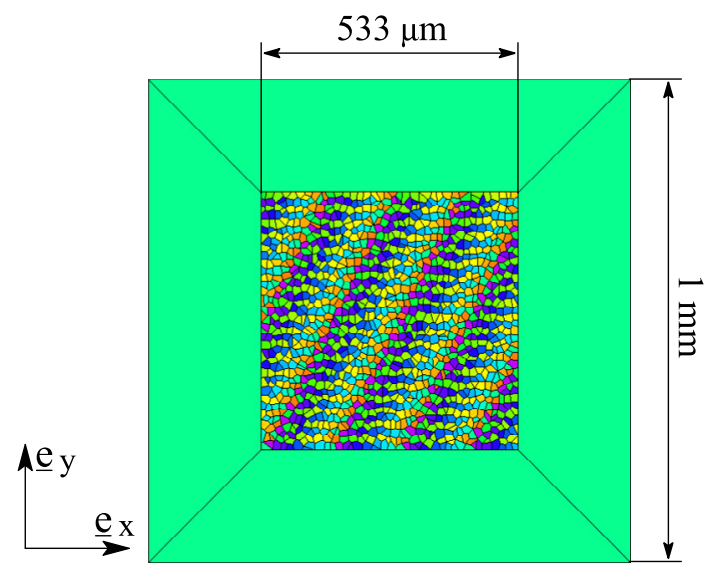

Fig. 9. Geometry of the smooth polycrystalline aggregate used to conduct FEA representative of conventional fatigue tests. 
viously discussed, is used to partition the geometry into subdomains. The resulting microstructure contains the same number of grains as the polycrystalline unit cell. These grains are discretized in average with 23 triangular FEs and their mechanical behavior is described by the constitutive model presented in Section 2.2.2. The polycrystalline aggregate is embedded in a homogeneous matrix in order to avoid applying the boundary conditions on the edges of the microstructure. The mechanical behavior assigned to this matrix is the same as the one used for the homogeneous stent (see Section 2.1). Uniform stress fields are applied to the edges of the matrix so that the boundary conditions are representative of stress-controlled fatigue tests. The polycrystalline aggregate is loaded at the average fatigue limit level in different loading conditions: fully reversed tension, fully reversed shear and tension with a loading ratio $R=0.1$. The average fatigue limits, defined at $2 \cdot 10^{6}$ cycles, has been estimated from fatigue tests conducted on a $316 \mathrm{~L}$ steel and reported in [27].

The distributions of the mesoscopic mechanical quantities obtained with polycrystalline unit cells and the smooth microstructures are presented in Fig. 10. The first, second, and third columns contain respectively the distributions of the shear stress amplitude $\tau_{a}$, of the normal stress amplitude $\sigma_{n, a}$ and of the mean normal stress $\sigma_{n, m}$. The diagrams in Fig. 10a correspond to the results obtained with the polycrystalline unit cells while the diagrams in Fig. 10b-d stem from the smooth microstructures loaded respectively in fully reversed tension, in fully reversed shear and in tension with a loading ratio $R=-1$.

From this figure, it appears clearly that the maximum values of the shear stress amplitude $\tau_{a}$ and the normal stress amplitude $\sigma_{n, a}$ steming from the polycrystalline unit cells are small in comparison with those obtained in smooth polycrystalline aggregates loaded in classical loading conditions in fatigue. Indeed, in the case of the polycrystalline unit cell, the shear stress amplitude $\tau_{a}$ and the normal stress amplitude $\sigma_{n, a}$ do not exceed respectively $20 \mathrm{MPa}$ and $45 \mathrm{MPa}$ whereas the case of fully reversed tension, for example, $\tau_{a}$ and $\sigma_{n, a}$ can reach respectively $135 \mathrm{MPa}$ and $324 \mathrm{MPa}$. On the contrary, the extremum values of the mean normal stress $\sigma_{n, m}$ observed in the unit cells are significantly larger than in the other loading cases, even in the case of the smooth microstructures loaded in tension with a loading ratio $R=0.1$. Indeed, the mesoscopic mean normal stress $\sigma_{n, m}$ can reach $790 \mathrm{MPa}$ in the unit cell whereas it does not exceed $260 \mathrm{MPa}$ in the case of tension with a loading ratio $R=0.1$. This large scatter of the mean normal stress $\sigma_{n, m}$ in the unit cell is due to the significant residual stress fields induced by the heterogeneous plastic slips occurring during the stent deployment.

To sum up, the stress states in the unit cells are far from those obtained in conventional fatigue tests and are characterized by:

- low amplitudes of shear stress $\tau_{a}$ and of normal stress $\sigma_{n, a}$;

- a large scatter of the mean normal stress $\sigma_{n, m}$;

- and significant stress gradients due to the stress raisers and to the strain incompatibilities between the grains.

\section{Fatigue criterion}

\subsection{Definition of the fatigue criterion}

As shown previously, the loading conditions imposed on the complex geometry of the stent unit cell lead to a stress gradient in the structure. In these conditions, it is of crucial importance to establish a fatigue design methodology reflecting the beneficial effect of a stress gradient on the fatigue strength. Indeed, it is commonly observed that applying a classical fatigue criterion on the critical point at the notch root leads to significant underestimations of the fatigue limit $[35,36]$.

Sheppard [37] suggested that the fatigue strength in terms of crack initiation and early growth may be dependent on the overall stress state in a finite volume in the vicinity of the stress concentration, as opposed to the stress state at a notch tip and thus proposed to consider, in the fatigue criterion, the average stress over a fatigue damage process zone (PZ) rather than the peak stress. In the case of notched components, Taylor proposed to define the fatigue damage process zone by a semi-circular area centered on the "hot spot", the critical point at the notch root [35]. The radius of the semi-circular area can be identified such as the prediction of the fatigue criterion is in accordance with the fatigue limit obtained from a notch specimen or defined by:

- a length related to some geometric characteristics of the microstructure (mean grain size, inclusion spacing...),

- the effective crack length $l_{0}$ introduced by El Haddad et al. [38], - empirical rules proposed by Atzori et al. [39].

Although this approach may be of undeniable practical interest for the fatigue design, it generally does not take into account the specificities of the microstructure. In particular, the variability of the microstructure in the process zone is neglected because the stress state in the surrounding of the notch is determined assuming a homogeneous linear elastic behavior. The predictions of the fatigue criterion are thus unable to reflect the scatter of the fatigue strength which might be induced by the lack of representativeness of the crystalline orientations in the critical region of a stress concentration.

An other approach, proposed by Owolabi et al. [40], provides a definition of the fatigue damage process zone based on a "fatigue indicator parameter" denoted $\Delta \Gamma$. This method consists in characterizing the susceptibility to fatigue crack initiation in each grain thanks to an equivalent mechanical quantity $\Delta \Gamma$ and to define the process zone with the grains whose $\Delta \Gamma$ values are greater or equal to a threshold value. This methodology provides a definition of a microstructure-dependent fatigue damage process zone.

The method proposed in this study is a compromise between these two approaches. The fatigue damage process zone is defined by a critical grain and its neighborhood, leading approximately to a circular area centered on the critical grain and related to the microstructural features of the material. Before explaining in detail the fatigue criterion, some remarks are necessary: the mechanical quantities used in the criterion are computed from the stress tensors averaged per grain $\langle\underline{\underline{\sigma}}\rangle_{g}$ which are obtained from the last loading cycle of the FE simulations. Moreover, the grains in contact with the edges where the boundary conditions are applied, are excluded from the analysis given that their stress states are in average overestimated with respect to those observed in the homogeneous stent.

In order to determine the critical grain with respect to the fatigue crack initiation, it is necessary to define a relevant fatigue indicator parameter. In ductile metallic alloys, the fatigue crack initiation is driven by the irreversible slip occuring in the grains. In these conditions, the amplitude of the shear stress $\tau_{a}$ acting on the slip planes is chosen to provide a approximate measure of the slip activity. Moreover, the fatigue crack initiation can be significantly affected by the normal stress acting on the slip plane. Indeed, a positive normal stress contributes to the decohesion at the slip band interface. Thus, it seems relevant to include this mechanical quantity in the fatigue criterion. More precisely, a distinction is made between the amplitude and the mean of the normal stress, respectively $\sigma_{n, a}$ and $\sigma_{n, m}$ (see [27] for the definitions of these mechanical quantities). 

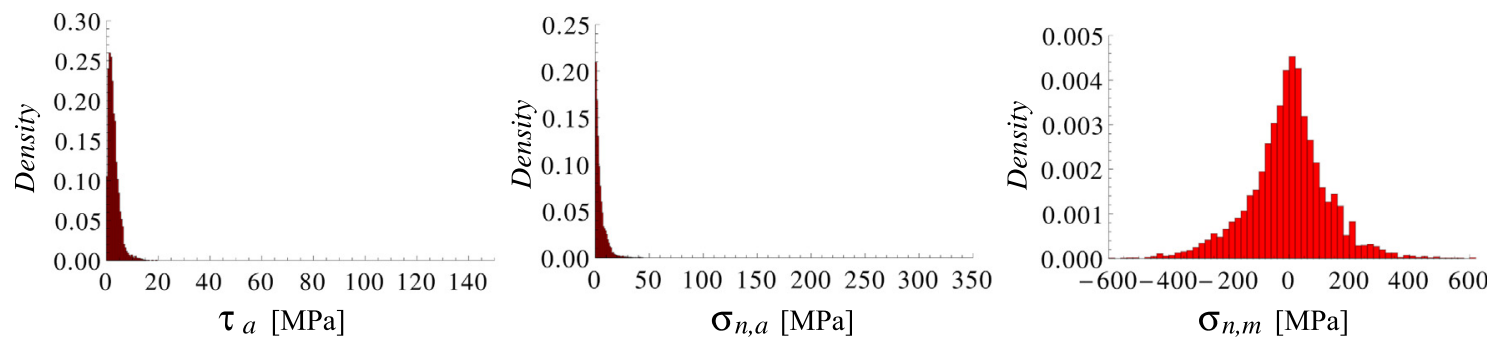

(a) Polycrystalline stent unit cells
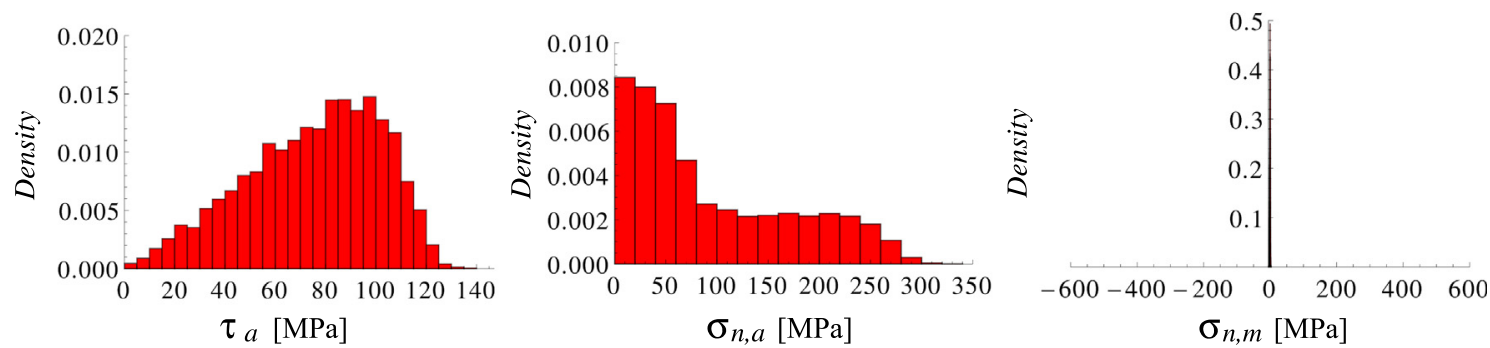

(b) Tension $\left(R_{\sigma}=-1\right)$
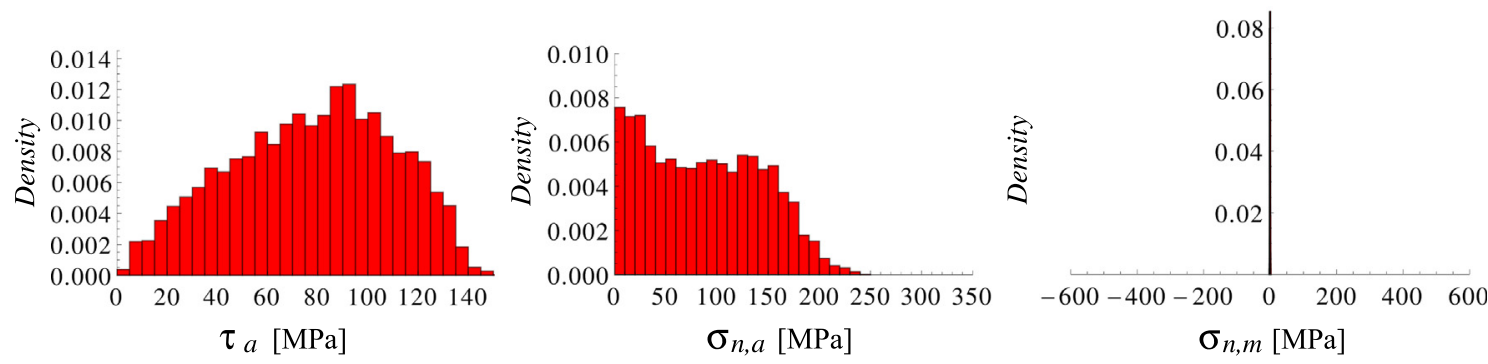

(c) Torsion $\left(R_{\sigma}=-1\right)$
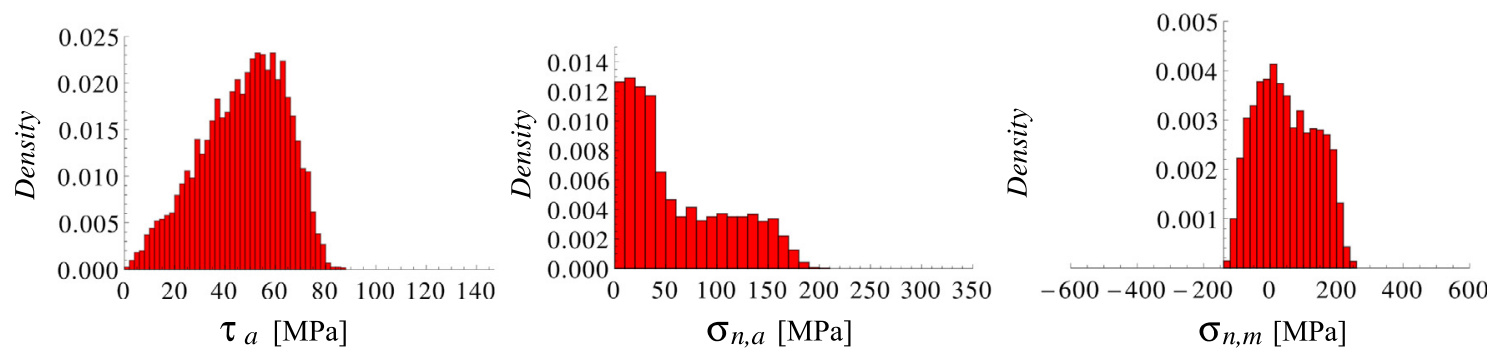

(d) Tension $\left(R_{\sigma}=0.1\right)$

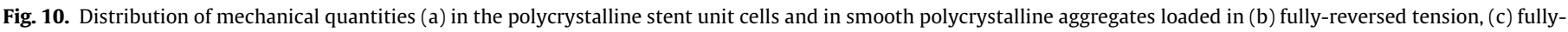
reversed shear and (d) in tension with $R_{\sigma}=0.1$.

The simplest formulation possible for the fatigue indicator parameter is a linear combination of the three aforementioned mechanical quantities. However, as each grain contains four slip planes, four values of this combination can be computed per grain. The maximum value among those associated to the slip planes of normal unit-vector $\underline{n}$ is chosen, leading to the following definition of fatigue indicator parameter $\sigma_{e q}$ :

$\sigma_{e q}=\max _{\underline{n}}\left[\tau_{a}(\underline{n})+\alpha \sigma_{n, a}(\underline{n})+\beta \sigma_{n, m}(\underline{n})\right]$

where $\alpha$ and $\beta$ are two material constant describing, respectively, the sensitivity of the fatigue limit to the mesoscopic normal stress amplitude $\sigma_{n, a}$ and to the mesoscopic mean normal stress $\sigma_{n, m}$. It should be noted that the present fatigue indicator parameter can be view as a transposition, at the mesoscale, of the damage indica- tor, defined at the macroscale, proposed by Vidal et al. [41] in a critical plane criterion.

In order to constitute the grain set defining the fatigue damage process zone $G_{\mathrm{PZ}}$, the critical grain has to be identified. This grain corresponds to the one having the maximum value of the fatigue indicator parameter $\sigma_{e q}$ among the grains composing the stent unit cell $G_{\text {cell }}$ :

$\underset{g \in G_{\text {cell }}}{\arg \max }\left[\sigma_{e q}\right]$

Once the critical grain $g_{\text {crit }}$ is determined for a given microstructure, the set of grains $G_{\mathrm{Pz}}$, defining the fatigue damage process zone and gathering $g_{\text {crit }}$ and the grains belonging to its $N_{n}$ first neighborhoods, is constructed:

$G_{\mathrm{PZ}}=\left\{g \in n\right.$-th neighborhood $\left.\mid n \leqslant N_{n}\right\}$ 
where $N_{n}$ corresponds to the number of neighborhoods considered in the fatigue damage process zone. A grain $g$ is considered in the neighborhood $n$ if at least one of its nodes is on the boundary of one of the grains in the neighborhood $n-1$. The neighborhood $n=0$ corresponds to the critical grain. In order to illustrate the construction of the grain set $G_{\mathrm{Pz}}$, an example of a critical grain and its neighborhoods is presented in Fig. 11. In this figure, the critical grain is highlighted in red and its first and second neighborhoods are colored respectively in blue and in yellow.

Finally, the fatigue failure of the stent unit cell is assumed to be prevented as long as the arithmetic mean of the fatigue indicator parameter $\sigma_{e q}$ weighted by the volume fraction $f_{g}$ of the grains does not exceed a threshold value $\gamma$ :

$\sigma_{e q, w}=\sum_{g \in G_{\mathrm{PZ}}}\left[f_{g} \sigma_{e q}\right] \leqslant \gamma$

with $f_{g}$ corresponding to the volume fraction of the grain $g$ in the grain set $G_{\mathrm{PZ}}$. In other words, the volume fraction $f_{g}$ is defined as the ratio between the volume of the grain $g$ and the volume of the fatigue damage process zone.

It is worth noting that, with this definition of the fatigue criterion, the process zone selected does not necessarily correspond to the set of grains leading to the highest value of the weighted equivalent stress $\sigma_{e q, w}$. Indeed, it is possible, in certain cases, to find a grain set giving a higher value of the weighted equivalent stress $\sigma_{e q, w}$ and for which the central grain is different than the critical grain. It would have been possible to define the process zone with the grain set leading to the highest $\sigma_{e q, w}$ value rather than the grain set for which the central grain correspond to the critical grain. Since none of these two definitions of the process zone seems more appropriate than the other, the one requiring the simplest implementation has been chosen.

\subsection{Calibration procedure}

The fatigue criterion has four parameters which have to be identified:

1. $N_{n}$, the number of neighborhoods considered in the fatigue damage process zone, which moderate the effect of a stress concentration on the fatigue strength;

2. $\alpha$ which tempers the detrimental effect of the mesoscopic normal stress amplitude $\sigma_{n, a}$ on the fatigue strength;

3. $\beta$ which describes the sensitivity of the fatigue limit to the mesoscopic mean normal stress $\sigma_{n, m}$;

4. $\gamma$, the fatigue crack initiation threshold.

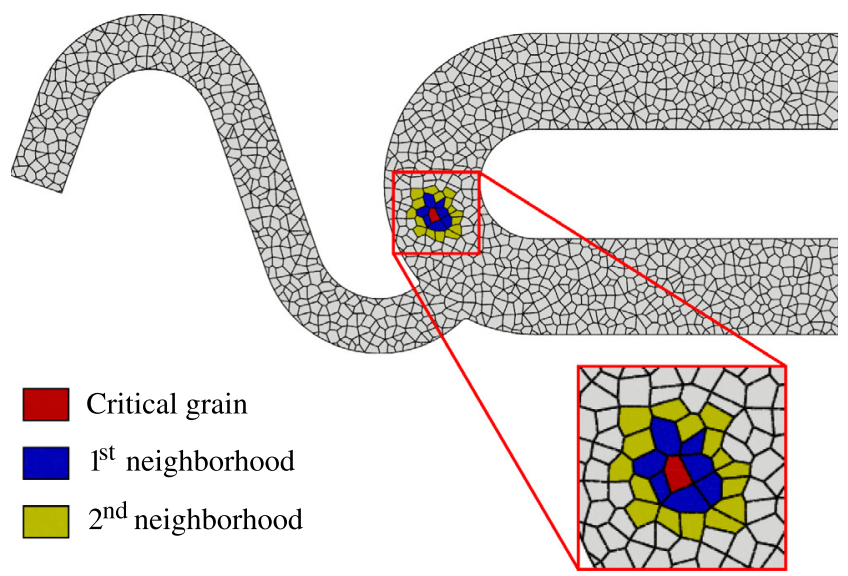

Fig. 11. Example of the critical grain and its 2 first neighborhoods in a stent unit cell.
The number of neighborhood $N_{n}$ is imposed and different values are considered: $0,1,2,3$ and 4 . The other parameters are calibrated for each value of $N_{n}$.

Contrary to classical fatigue criteria applied at the macroscopic scale (see for example [42-45]), these parameters cannot be related explicitly to conventional average macroscopic fatigue limits. Indeed, the determination of closed-form expressions is not possible due to complex distributions of the mechanical quantities at the mesoscopic scale, even in the case of smooth microstructures with homogeneous loading conditions. Thus the calibration procedure requires the use of FE simulations of polycrystalline aggregates.

The strategy adopted in the present work consists in conducting FEA representative of conventional fatigue tests. In this purpose, the FE models with smooth microstructures, presented in Section 3, are used. The geometry of the microstructure, presented in Fig. 9 contains the same number of grains as the unit cell. Twenty different orientation sets are used allowing to investigate, as in the case of the stent unit cell, twenty realizations of microstructures. The polycrystalline aggregates are loaded at the average fatigue limit level in fully reversed tension, in fully reversed shear and in tension with a loading ratio $R=0.1$. These loading conditions have been selected for their simplicity and the diversity of the stress states they induce at the mesoscopic scale. More precisely, the tension with a positive loading ratio $R$ emphasizes the mesoscopic mean normal stress $\sigma_{n, m}$ allowing the calibration of the parameter $\beta$ whereas the fully reversed tension and the fully reversed torsion induce different ratios $\tau_{a} / \sigma_{n, a}$ allowing to identify $\alpha$ and $\gamma$.

For each value of the parameter $N_{n}$, the parameters $\alpha, \beta$ and $\gamma$ are identified such that the weighted equivalent stress $\sigma_{e q, w}$ is, in average on the 20 different realizations of microstructures, equal to $\gamma$ for each of the three loading conditions (Eq. (19)). The different sets of parameters obtained are summarized in Table 4 .

$$
\left\langle\sigma_{e q, w}\right\rangle_{m}=\frac{1}{N_{m}} \sum_{m=1}^{N_{m}}\left[\sigma_{e q, w}\right]=\gamma
$$

In order to illustrate the influence of the number of neighborhoods on the predictions of the criterion, the latter is applied on microstructures exhibiting a stress concentration. The geometry of the polycrystalline aggregates is similar to the one presented in Fig. 9 excepted that it contains a circular hole with a diameter $D=95 \mu \mathrm{m}$ centered in the microstructures. These polycrystalline aggregates are loaded in fully reversed tension. The macroscopic normal stress amplitude $\Sigma_{x x, a}$ applied on the matrix corresponds to the average macroscopic fatigue limit and is equal to $213 \mathrm{MPa}$ (see [27]). For each set of parameters, the ratio $\left\langle\sigma_{e q, w}\right\rangle_{m} / \gamma$ is evaluated and the predictions are presented in Table 5.

As the microstructures are loaded at the average macroscopic fatigue limit level, the fatigue criterion accurately predicted the influence of the stress gradient on the fatigue strength if the ratio $\left\langle\sigma_{e q, w}\right\rangle_{m} / \gamma=1$. If the value of this ratio is greater than 1 , the detrimental effect of the stress concentration on the fatigue limit is overestimated. It is worth noting that considering first four neighborhoods in the fatigue damage process zone leads to a correct pre-

Table 4

Fatigue criterion parameters identified for a 316L steel.

\begin{tabular}{llll}
\hline$N_{n}$ & $\alpha$ & $\beta$ & $\gamma(\mathrm{MPa})$ \\
\hline 0 & 0.193 & 0.317 & 163 \\
1 & 0.194 & 0.312 & 132 \\
2 & 0.194 & 0.323 & 129 \\
3 & 0.194 & 0.318 & 127 \\
4 & 0.206 & 0.317 & 128 \\
\hline
\end{tabular}


Table 5

Predictions of the ratio $\left\langle\sigma_{e q, w}\right\rangle_{m} / \gamma$ with respect to the number of neighborhood considered in the fatigue damage process zone.

\begin{tabular}{llllll}
\hline$N_{n}$ & 0 & 1 & 2 & 3 & 4 \\
\hline$\left\langle\sigma_{e q, w}\right\rangle_{m} / \gamma$ & 1.39 & 1.36 & 1.22 & 1.11 & 1.01 \\
\hline
\end{tabular}

diction of the influence of the stress gradient induced by the hole on the fatigue strength.

\section{Probabilistic analysis of the fatigue criterion predictions}

The weighted equivalent stress $\sigma_{e q, w}$ defined in Eq. (18) is a continuous random variable whose value depends on the crystalline orientations assigned to the grains. Thus, rather than predicting deterministically the fatigue crack initiation for specific realizations of stent unit cells, it could be more interesting to place the fatigue design of stents in a probabilistic framework and thus to directly permit the prediction of the survival probability of the stent unit cell $P_{S, \text { cell }}$.

This probability can be roughly estimated by the empirical survival probability of the unit cell which is determined by comparing the weighted equivalent stress $\sigma_{e q, w}$ of each realization of microstructure with the fatigue crack initiation threshold $\gamma$ and by computing the ratio of the number of surviving unit cells divided by the total number of unit cells. The main disadvantage of this method arises when the estimated probability is either very close to 0 or to 1 . It's important to emphasize that this situation can occur since the failure probability aimed, for a suitable fatigue design, is generally ranging from 0 to $10^{-6}$. In this case, a very large sample of microstructures is required in order to accurately estimate the survival probability. However, due to significant computation time, only a limited number of realizations of microstructures can be studied. In these conditions, it could be more relevant to describe the random variable $\sigma_{e q, w}$ with a continuous probability distribution in order to evaluate the survival probability of the unit cell of the stent.

Several continuous probability distributions have been tested in order to adequately approximate the distribution from which the set of $\sigma_{\text {eq.w }}$ is sampled. Due to the asymmetry of the distribution of the weighted equivalent stress $\sigma_{e q, w}$ (represented by a histogram in Fig. 12), the following asymmetric probability distributions have been considered: the Weibull distribution with or without location parameter, the Gumbel distribution and the Frechet distribution. The parameters of these distributions are estimated using the method of the maximum likelihood estimation. The best fit is

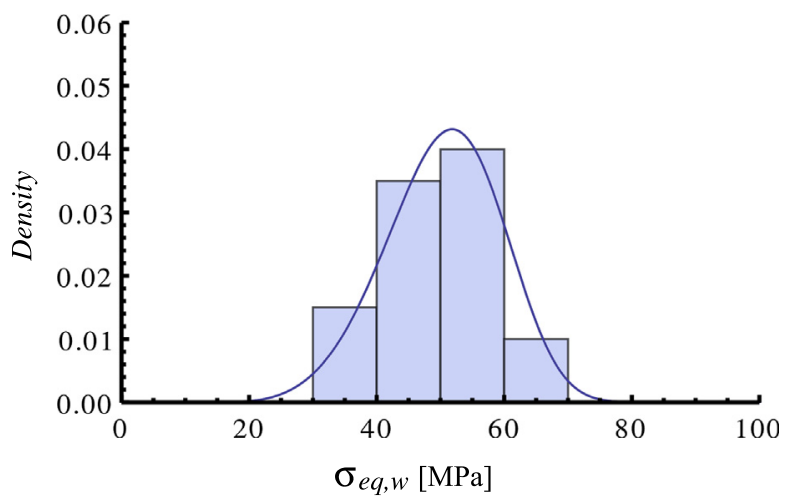

Fig. 12. Empirical distribution of the weighted equivalent stress $\sigma_{e q, w}$ represented by a histogram and probability density function $f$ of the Weibull distribution. obtained in the case of the Weibull distribution using the location parameter, defined by the following equation:

$f\left(\sigma_{e q, w}\right)= \begin{cases}\frac{k}{\lambda}\left(\frac{\sigma_{e q, w}-\theta}{\lambda}\right)^{k-1} \exp \left[-\left(\frac{\sigma_{e q, w}-\theta}{\lambda}\right)^{k}\right] & \text { if } \sigma_{e q, w} \geqslant \theta \\ 0 & \text { if } \sigma_{e q, w}<\theta\end{cases}$

where $k, \lambda$ and $\theta$ correspond respectively to the shape, scale and location parameters. The resulting probability density function is plotted, in addition to the empirical distribution of the weighted equivalent stress $\sigma_{\text {eq,w }}$, in the diagram presented in Fig. 12. The cumulative distribution function $F$ of the Weibull distribution is expressed as follows:

$F\left(\sigma_{e q, w}\right)= \begin{cases}1-\exp \left[-\left(\frac{\sigma_{e q, w}-\theta}{\lambda}\right)^{k}\right] & \text { if } \sigma_{e q, w} \geqslant \theta \\ 0 & \text { if } \sigma_{e q, w}<\theta\end{cases}$

The survival probability of a stent unit cell $P_{\text {s.cell, }}$, which corresponds to the probability that the weighted equivalent stress $\sigma_{\text {eq.w }}$ is lower than the fatigue crack initiation threshold $\gamma$, can be simply determined from the cumulative distribution function $F$ :

$P_{s, \text { cell }}=P\left(\sigma_{e q, w} \leqslant \gamma\right)=F(\gamma)$

In order to determine the survival probability of a complete stent $P_{S \text { stent }}$, knowing the survival probabilities of the stent unit cells $P_{\text {S.cell }}$ constituting the stent, the weakest link hypothesis is used, leading to the following equation:

$P_{S, \text { stent }}=\prod_{\text {cell } \in C} P_{S, \text { cell }}$

It is worth noting that the choice of the weakest link hypothesis is appropriate in the present case because the random variables are independent. Indeed, the value of the weighted equivalent stress $\sigma_{\text {eq.w }}$ in a stent unit cell does not affect the one obtained in an other unit cell.

As previously discussed, the mechanical response in the homogeneous stent is not exactly identical from one unit cell to the other. So, in order to properly evaluate the survival of the complete stent, it is necessary to determine the survival probability of each unit cell. However, due to the 6-fold rotational symmetry, about the $Z$-axis, of the structure and of the boundary conditions, the stent unit cells located in the same interval $\left[z_{\min }, z_{\max }\right]$ have the same survival probability $P_{S, \text { cell }}$. Thus, the survival probabilities of only 8 different unit cells are needed to determine the survival of the complete stent $P_{S, s t e n t}$. The evaluation of the survival probability of a given unit cell requires to apply on the polycrystalline unit cell, the boundary conditions steming from the considered unit cell of the homogeneous stent. However, due to large computation times, only the unit cell exhibiting the maximum von Mises stress has been studied in the present work. The survival probability of each unit cell are thus assumed to be equal to the one associated to the unit cell studied. This assumption seems reasonable because the differences between the mechanical responses of the different unit cells are small. As a result, the survival probability of the complete stent becomes:

$P_{S, \text { stent }}=P_{S, \text { cell }}^{N_{c}}$

where $N_{c}$ corresponds to the number of unit cells constituting the stent. In the present case, $N_{c}=48$

Both the survival probability of the unit cell $P_{S, \text { cell }}$ and the survival probability of the stent have been evaluated $P_{S, \text { stent }}$ for different loading conditions. More precisely, in order to study the effect of the arterial pressure amplitude $P_{a}$ on the survival probabilities of the unit cell and of the stent, different values of amplitude $P_{a}$ has been considered: 3, 6, 9 and $12 \mathrm{kPa}$. Different numbers of neighborhoods $N_{n}(0,1,2$ and 4$)$ have been chosen in the fatigue criterion in 


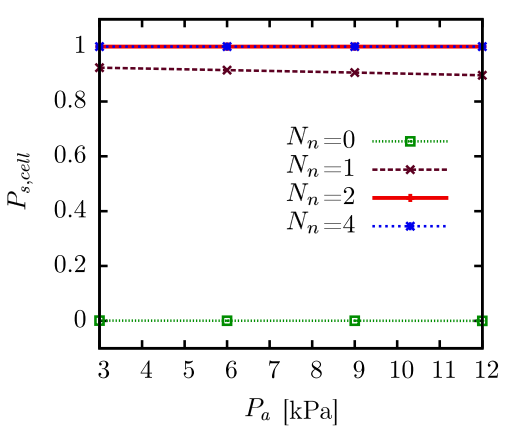

(a)

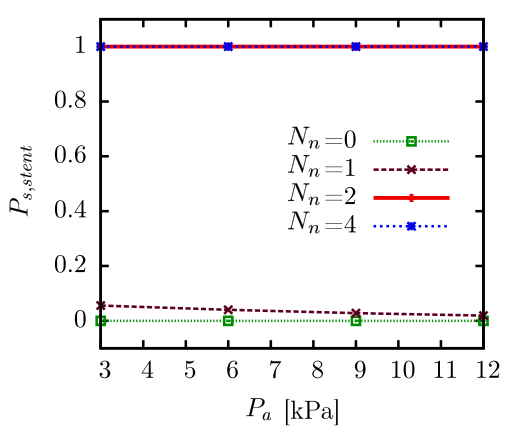

(b)

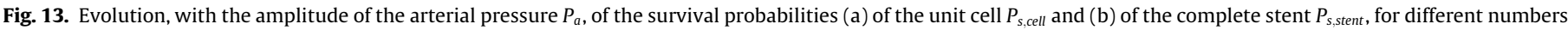
of neighborhoods $N_{n}$.

order to illustrate the effect of this parameter on the predictions of the fatigue criterion. However, it is recalled that the correct number of neighborhood $N_{n}$ which have to be considered is 4 according to the calibration procedure discussed in Section 4.2. The predicted survival probabilities $P_{S, \text { cell }}$ and $P_{S, \text { stent }}$ are presented respectively in Fig. 13a and b. It is worth noting that when the number of neighborhoods $N_{n}=4$, the survival probability of the complete stent $P_{s, \text { stent }}$ is equal to 1 and is thus in accordance with the observation of Kapnisis et al. [24]. Indeed, in the loading condition tested by the authors $\left(P_{a}=6 \mathrm{MPa}\right)$, no fatigue crack initiation was noted in the 316L Cypher ${ }^{\circledR}$ stent. Nonetheless, in order to properly evaluate the ability of the fatigue criterion to predict the survival probability of the complete stent, more experimental data are required. In particular, it could be interesting to carry out high-cycle fatigue tests in other loading conditions, especially loading conditions for which the survival probability is different than 1 .

From Fig. 13, it can also be observed that, when the number of neighborhoods $N_{n} \geqslant 2$, the effect of the arterial pressure amplitude $P_{a}$ on the survival probabilities of the unit cell $P_{\text {s.cell }}$ and of the stent $P_{\text {s.stent }}$ is negligible for the range of amplitude $P_{a}$ considered. Indeed, both the survival probabilities of the unit cell $P_{s, \text { cell }}$ and of the stent $P_{s, \text { stent }}$ are equal to 1 . No influence of the arterial pressure amplitude $P_{a}$ on the probabilities $P_{s, \text { cell }}$ and $P_{s, s t e n t}$ can also be observed when $N_{n}=0$ but the predicted survival probabilities are equal 0 in this case. The influence is only perceptible when the number of neighborhoods $N_{n}=1$. In this condition, an increase in the amplitude of the arterial pressure $P_{a}$ leads to a slight decrease in the survival probabilities $P_{\text {s,cell }}$ and $P_{s, \text { stent }}$. Moreover, it is worth noting that, even though the survival probability of the unit cell $P_{s, \text { cell }}$ is high (close to 0.9), the survival probability of the complete stent $P_{s, s t e n t}$ becomes significantly small (lower than 0.1 ).

\section{Conclusions}

The present paper has proposed a methodology for the fatigue design of polycrystalline structures which has been applied to a balloon-expandable stent made of $316 \mathrm{~L}$ austenitic steel. The approach relies on numerical simulations of polycrystalline stent unit cells enabling to study the mechanical responses at the grain scale. A comparison between the results obtained with the stent unit cells and those stemming from smooth microstructures subjected to loading conditions representative of classical fatigue tests has allowed to highlight the noteworthy characteristics of the mechanical response in the stent: the extremum value of the mean normal stress $\sigma_{n, m}$ are significant whereas the amplitude of both the shear stress $\tau_{a}$ and the normal stress $\tau_{n, a}$ are small. Another feature observed was the notable heterogeneity of the mechanical fields in the stent unit cells which can be attributed to:
- the stress concentrations in the unit cell geometry,

- the strain incompatibilities between the grains induced by the anisotropy of their mechanical behavior.

A fatigue criterion, suited to structures subjected to stress gradients and using the stress states at the grain scale, has been proposed and applied to several realizations of microstructures. The equivalent stress introduced in this criterion is sensitive to the microstructure and has been described by a continuous probability distribution in order to estimate the survival probability of the stent unit cell. Thanks to some assumptions (e.g. the weakest link hypothesis), the predictions have been extended to the complete stent. The predicted survival probability of the stent is in accordance with the experimental data from the literature [24]. Moreover, the influence of the amplitude of the arterial pressure on the fatigue strength of the stent has been evaluated. It has appeared that a variation of the arterial pressure amplitude $P_{a}$ from 3 to $12 \mathrm{kPa}$ does not noticeably affect the survival probability of the stent predicted by the fatigue criterion when the number of neighborhoods obtained with the calibration $\left(N_{n} \geqslant 4\right)$ is used.

Although the methodology developed in this work is promising, it is worth noting that the modeling of the polycrystalline stent relies on strong assumptions (e.g. the stent crimping has been neglected in the FEA of the three-dimensional model, the stent unit cell is modeled by a two-dimensional geometry, the initial residual stress field introduced by the manufacturing process has been neglected). Moreover, this methodology has not yet been validated for loading conditions leading to survival probabilities different to 1. Thus, further investigations are needed to assess thoroughly the predictive ability of the fatigue criterion. For this purpose, it could be interesting to carry out an exhaustive fatigue test series in order to estimate experimentally the survival probabilities of a specimen under different loading conditions and to compare them to the survival probabilities predicted with the proposed methodology. In particular, selecting tests with survival probabilities different to 1 and validating the fatigue criterion for these loading conditions are essential to ensure that the proposed methodology is a relevant fatigue design tool.

Furthermore, the only source of fatigue strength scatter discussed in the present paper is stemming from the variability of the mesoscopic mechanical quantities induced by the change of crystalline orientations. However, we recall that other causes, not investigated in this work, can contribute to fatigue scatter such as the variability of the:

- stent geometry which is related to the repeatability of the manufacturing process,

- other microstructural atributes (grain size [18], grain size distribution [46], precipitate [18]), 
- loading conditions applied on the stent which depend on the geometry and the mechanical behavior of the vessel and of the atherosclerotic plaque.

These sources of variability would deserve to be studied in order to determine those which influence prominently the scatter of the fatigue strength and to refine the fatigue design methodology accordingly.

\section{Acknowledgments}

This work is funded by the French National Research Agency (Project Fast3D-ANR-11-BS09-012-01) and by the Fondazione Cariplo(Grant 2013-1779). The authors would like to acknowledge Dr. Michele Conti for his useful advice and for providing the threedimensional stent mesh used in the present work.

\section{References}

[1] AL-Mangour B, Mongrain R, Yue S. Coronary stents fracture: an engineering approach (review). Mater Sci Appl 2013;4(10):606-21.

[2] Aoki J, Nakazawa G, Tanabe K, Hoye A, Yamamoto H, Nakayama T, Onuma Y, Higashikuni Y, Otsuki S, Yagishita A, Yachi S, Nakajima H, Hara K. Incidence and clinical impact of coronary stent fracture after sirolimus-eluting stent implantation. Catheterization Cardiovasc Interventions 2007;69(3):380-6.

[3] Adlakha S, Sheikh M, Wu J, Burket M, Pandya U, Colyer W, Eltahawy E, Cooper C. Stent fracture in the coronary and peripheral arteries. J Interventional Cardiol 2010;23(4):411-9.

[4] Smouse H, Nikanorov A, LaFlash D. Biomechanical forces in the femoropopliteal arterial segment what happens during extremity movement and what is the effect on stenting? Endovasc Today 2005:4(6):60-6.

[5] Wiersma S, Dolan F, Taylor D. Fatigue and fracture in materials used for microscale biomedical components. Biomed Mater Eng 2006;16(2):137-46.

[6] Weiss S, Meissner A, Fischer A. Microstructural changes within similar coronary stents produced from two different austenitic steels. J Mech Behav Biomed Mater 2009;2(2):210-6.

[7] International standard ISO 25539-2 Cardiovascular implants - endovascular devices - Part 2: Vascular stents; 2012.

[8] US Food, Drug Administration, Non-clinical engineering tests and recommended labeling for intravascular stents and associated delivery systems: guidance for industry and FDA staff. US Department of Health and Human Services, Food and Drug Administration, Centre for Devices and Radiological, Health; April 18th 2010.

[9] Auricchio F, Constantinescu A, Conti M, Scalet G. Fatigue of metallic stents: from clinical evidence to computational analysis. Ann Biomed Eng 2015:1-15.

[10] McGarry J, O'Donnell B, McHugh P, McGarry J. Analysis of the mechanical performance of a cardiovascular stent design based on micromechanical modelling. Comput Mater Sci 2004;31:421-38.

[11] Marrey RV, Burgermeister R, Grishaber RB, Ritchie R. Fatigue and life prediction for cobalt-chromium stents: a fracture mechanics analysis. Biomaterials 2006;27(9):1988-2000.

[12] Harewood F, McHugh P. Modeling of size dependent failure in cardiovascular stent struts under tension and bending. Ann Biomed Eng 2007;35(9):1539-53.

[13] Li J, Luo Q, Xie Z, Li Y, Zeng Y. Fatigue life analysis and experimental verification of coronary stent. Heart Vessels 2010;25(4):333-7.

[14] Azaouzi M, Makradi A, Belouettar S. Fatigue life prediction of cardiovascular stent using finite element method. Comput Methods Biomech Biomed Eng 2012;15(S1):93-5.

[15] dos Santos H, Auricchio F, Conti M. Fatigue life assessment of cardiovascular balloon-expandable stents: a two-scale plasticity-damage model approach. J Mech Behav Biomed 2012;15:78-92.

[16] Barrera O, Makradi A, Abbadi M, Azaouzi M, Belouettar S. On high-cycle fatigue of 316L stents. Comput Methods Biomech Biomed Eng 2012;0:1-12.

[17] Sweeney CA, McHugh PE, McGarry JP, Leen SB. Micromechanical methodology for fatigue in cardiovascular stents. Int J Fatigue 2012;44:202-16.

[18] Sweeney C, O’Brien B, Dunne F, McHugh P, Leen S. Strain-gradient modelling of grain size effects on fatigue of cocr alloy. Acta Mater 2014;78(0):341-53.

[19] Sweeney C, O'Brien B, McHugh P, Leen S. Experimental characterisation for micromechanical modelling of $\mathrm{CoCr}$ stent fatigue. Biomaterials 2014;35:36-48.
[20] Sweeney C, O'Brien B, Dunne F, McHugh P, Leen S. Micro-scale testing and micromechanical modelling for high cycle fatigue of cocr stent material. J Mech Behav Biomed Mater 2015;46(0):244-60.

[21] Auricchio F, Constantinescu A, Conti M, Scalet G. A computational approach for the lifetime prediction of cardiovascular balloon-expandable stents. Int J Fatigue 2015;75:69-79.

[22] Dordoni E, Petrini L, Wu W, Migliavacca F, Dubini G, Pennati G. Computational modeling to predict fatigue behavior of niti stents: what do we need? J Functional Biomater 2015;6(2):299.

[23] Auricchio F, Constantinescu A, Scalet G. Fatigue of 316L stainless steel notched um-size components. Int J Fatigue 2014;68:231-47.

[24] Kapnisis K, Constantinides G, Georgiou H, Cristea D, Gabor C, Munteanu D, Brott B, Anderson P, Lemons J, Anayiotos A. Multi-scale mechanical investigation of stainless steel and cobalt-chromium stents. J Mech Behav Biomed Mater 2014;40:240-51.

[25] Guerchais R. Influence d'accidents géométriques et du mode de chargement sur le comportement en fatigue à grand nombre de cycles d'un acier inoxydable austénitique 316L. Ph.D. thesis, Art et Métiers ParisTech; 2014.

[26] Makino A, Hamburger W, Fitch J. Fluoroelastomer pressure pad design for microelectronic applications. Tech. rep., Western Research Laboratory Research Report 93/7; 1993.

[27] Guerchais R, Morel F, Saintier N, Robert C. Influence of the microstructure and voids on the high-cycle fatigue strength of $316 \mathrm{~L}$ stainless steel under multiaxial loading. Fatigue Fract Eng Mater Struct 2015;38:1087-104.

[28] Castelluccio GM, McDowell DL. Effect of annealing twins on crack initiation under high cycle fatigue conditions. J Mater Sci 2012;48(6):2376-87.

[29] Teklu A, Ledbetter H, Kim S, Boatner L, McGuire M, Keppens V. Single-crystal elastic constants of fe-15ni-15cr alloy. Metall Mater Trans A 2004;35 (10):3149-54.

[30] Méric L, Cailletaud G, Gaspérini M. F.E. calculations of copper bicrystal specimens submitted to tension-compression tests. Acta Metall Mater 1994;42(3):921-35.

[31] Franciosi P. étude théorique et expérimentale du comportement élastoplastique des monocristaux métalliques se déformant par glissement: modélisation pour un chargement complexe quasi statique. Ph.D. thesis, Université Paris 13; 1984.

[32] Eisenlohr P, Roters F. Selecting a set of discrete orientations for accurate texture reconstruction. Comput Mater Sci 2008;42(4):670-8.

[33] Fortune S. A sweepline algorithm for voronoi diagrams. Algorithmica 1987;2 $(1-4): 153-74$

[34] Geuzaine C, Remacle J-F. Gmsh: A 3-D finite element mesh generator with built-in pre- and post-processing facilities. Int J Numer Methods Eng 2009;79 (11):1309-31.

[35] Taylor D. Geometrical effects in fatigue: a unifying theoretical model. Int J Fatigue 1999;21(5):413-20.

[36] Bertolino G, Constantinescu A, Ferjani M, Treiber P. A multiscale approach of fatigue and shakedown for notched structures. Theor Appl Fract Mech 2007;48 (2):140-51.

[37] Sheppard SD. Field effects in fatigue crack initiation: long life fatigue strength. Trans ASME 1991;113(2):188-94.

[38] El Haddad M, Smith K, Topper T. Fatigue crack propagation of short cracks. J Eng Mater Technol 1979;101(1):42-6.

[39] Atzori B, Meneghetti G, Susmel L. Material fatigue properties for assessing mechanical components weakened by notches and defects. Fatigue Fract Eng Mater Struct 2005;28(1-2):83-97.

[40] Owolabi G, Egboiyi B, Shi L, Whitworth H. Microstructure-dependent fatigue damage process zone and notch sensitivity index. Int J Fract 2011;170 (2):159-73.

[41] Vidal E, Kenmeugne B, Robert JL, Bahuaud J. Fatigue life prediction of components using multiaxial criteria. In: Pineau A, Cailletaud G, Lindley TC, editors. Multiaxial fatigue and design. Mechanical Engineering Publications; 1996. p. 365-78

[42] Crossland B. Effect of large hydrostatic pressures on the torsional fatigue strength of an alloy steel. In: Proc. int. conf. on fatigue of metals. Institution of Mechanical Engineers; 1956. p. 138-49.

[43] Sines G. Behaviour of metals under complex static and alternating stresses. In: Sines G, Waisman JL, editors. Metal fatigue. McGraw Hill; 1959. p. 145-69.

[44] Matake T. An explanation on fatigue limit under combined stress. Bull JSME 1977;20(141):257-63.

[45] Papadopoulos IV, Davoli P, Gorla C, Filippini M, Bernasconi A. A comparative study of multiaxial high-cycle fatigue criteria for metals. Int J Fatigue 1997;19 (3):219-35.

[46] Baudoin P, Magnier V, El Bartali A, Witz J-F, Dufrenoy P, Demilly F, et al. Numerical investigation of fatigue strength of grain size gradient materials under heterogeneous stress states in a notched specimen. Int J Fatigue 2016;87:132-42. 